\title{
Representation and Extension in Consciousness Studies
}

\author{
Zsuzsanna Kondor \\ Institute of Philosophy, \\ Research Centre for the Humanities, \\ Hungarian Academy of Sciences \\ kondorzs@gmail.com
}

Received 31 August 2016; accepted 25 July 2017.

\begin{abstract}
Various theories suggest conscious phenomena are based exclusively on brain activity, while others regard them as a result of the interaction between embodied agents and their environment. In this paper, I will consider whether this divergence entails the acceptance of the fact that different theories can be applied in different scales (as in the case of physics), or if they are reconcilable. I will suggest that investigating how the term representation is used can reveal some hints, building upon which we can bridge the gulf between the two poles in the long run. In my argumentation I will rely on some earlier philosophical insights, such as those of Descartes, James, Wittgenstein and Merleau-Ponty, as well as research based on global workspace theory, and the conceptions of embodied and enacted cognition. I will suggest that within a wider horizon of investigation, the ambiguity as regards the term representation will decrease.
\end{abstract}

Keywords: representation, consciousness, embodied cognition, sensorimotor approach. 
The phrase 'in the mind' has caused more confusion than almost any other in philosophy.

(Wittgenstein, 1979, p. 114)

\section{Introduction}

Mind, cognition, consciousness, and self have been regarded as closely related, both historically and functionally. ${ }^{1}$ However, their relationship is far from unambiguous. I will examine criticisms that call attention to misinterpretations of certain scientific results (Aizawa, 2010; Zahavi, 2008). These criticisms underscore the differences between certain perspectives and presuppositions. I will suggest that these presuppositions and perspectives may conceal, unnoticed, differences in the usage of some key terms, hence widening the gap between different approaches.

The term consciousness has numerous meanings, ${ }^{2}$ which presupposes various approaches. In certain aspects these approaches overlap and at the same time are divergent in some details. For example, despite the fact that phenomenology embraces and even relies on scientific evidence and, significantly, gives space to representationalism, it regards consciousness and the self in a radically different light than cognitive science. Similarly, proponents of embodiment and enaction embrace scientific results, but because of their commitment to embodiment (which is to some extent common with phenomenology) and in some cases explicit preference for an anti-representationalist setting, their comprehension of certain key concepts differs fundamentally with that of cognitive science.

If we give credit to the evidence which Gallagher (2005) and Jacob \& Jeannerod (2003) refer to when emphasizing the importance of the body, bodily skills, and activity, it may seem strange to query the role the body plays in our cognitive life. Also, considering the distinction between the minimal and the narrative self (Gallagher, 2000) in light of Ramachandran's (2004) effort to give a neural, brain-based account of linguistic and conceptual skills, it seems peculiar to regard the narrative self as mere abstraction (Dennett, 1991) since the capabilities which Ramachandran establishes on neural settings are hardly imaginable without interaction with others, i.e., without the extension of neural activity to bodily skills, which are public.

In this paper I will suggest that if we take a closer glance at the term representation, we will see considerable differences between various fields and authors regarding what we should have in mind when using this term. Accordingly, if we can illuminate the diverse meanings of representation, we may have a chance to bridge the gap between the theories that argue for the primordial role of the brain regarding consciousness and those which suggest that the body and the encompassing environment also play an important role when consciousness enters the scene. That is, I will suggest that going beyond cranial processes

\footnotetext{
${ }^{1}$ Consider the long history of the evolution of the term "consciousness" from Cudworth, via Locke, to phenomenology and more recently, research in psychology, cognitive neurology, and philosophy.

${ }^{2}$ Keeping in mind the difficulty of polymorphism and, from our present perspective more importantly, the difference in scope when scientific and philosophical theories are in question.
} 
is not calling the role of neuronal activity in conscious processes into question, but rather it opens up the possibility of reconsidering causal and representational relations. We can reconcile the represenationalism of cognitive science with approaches that endorse embodiment; thus it will be apparent that an extension of the cognitive system is not only necessary since representationalist accounts of neuronal and cognitive functions take into account the interaction with the body - at least on the neuronal level—but also because some obstacles are rooted in the ambiguous use of the term representation.

In the first section of the present paper, as a preliminary, I will consider a number of methodological difficulties including some meta-philosophical considerations. In the subsequent section I will attempt to give a rough outline of how consciousness emerges in scientific literature as focused on intracranial coherencies. In the third section I will outline some ideas that suggest widening the scope of investigation, extending it into bodily and environmental aspects of intelligent behaviour in accordance with a holistic and non-dualist approach. In the section after that, I will focus on the divergent ways representation is used in the literature and the questions that are concealed due to this confusion. In conclusion, I will attempt to briefly reconcile the neuronal-based and the rather holistic approaches to consciousness.

\section{Aims, Methods, Perspectives}

We must keep in mind the obstacles when attempting to take into consideration different kinds of approaches: some are considered as being so-called hard science (such as physics, and later, with the discovery of DNA, biology, and recently structural brain imaging), some as being soft science (we can have psychology in mind; Frith, 2007), and some as philosophical, since they are mostly regarded as being speculative. Nonetheless, it is an important enterprise. Despite this unfortunate constellation of diverging approaches, various applied methods, and convictions regarding measurements' objectivity vs. subjectivity, philosophy can hardly avoid taking into consideration what hard and soft sciences suggest, particularly in the case of cognitive capabilities and consciousness studies (Watson, 1995, p. 101).

Undoubtedly we need results produced by the sciences, but at the same time we have to be cautious of how we interpret them and investigate whether the scope of the evidence is not overestimated. When attempting to clarify the relation between the self and self-awareness, Dan Zahavi illuminated the dangers of thought experiments and, more importantly from our perspective, the difficulties we face when we try to interpret pathological cases. On the one hand, the interpretation of different pathological phenomena "usually depends on the framework within which one is operating," and on the other hand, "it is by no means clear what type of conclusion one should draw from" these cases (2008, p. 142). It is not clear whether pathological occurrences can be considered signs of anomaly within the theory, or mere exceptions. Do they reveal some hidden functions as regards normal behaviour, or show a compensatory mechanism entailed by a dysfunction? While we can in a more general manner say that "empirical data are important," their interpretation is up to the framework within which they are interpreted, hence "the theoretical impact of an empirical case is not necessarily something that can be easily determined” (Gallagher \& Zahavi, 2008, p. 221). 
Kenneth Aizawa recalls an experiment with Sur's rewired ferrets and reveals that it does not provide evidence for the idea propagated by some theorists such as Alva Noë, who suggests "that scientists should search for a broader biological correlate of consciousness" instead of focusing on intracranial neuronal processes (Aizawa, 2010b, p. 263).

The principal point to take away from this research is that Sur and his colleagues performed certain surgical interventions on newborn ferrets, then raised these ferrets to adulthood in the anticipation that these neonatal interventions will lead to differences in adult area A1. These anticipations have been borne out with Sur and his colleagues documenting the various ways in which rewired ferret A1 comes to resemble normal ferret V1. What these experiments suggest, contrary to Noë's view, is that when we look at the intrinsic features of the brain for a mechanistic explanation of the visual character of experience, we are probably looking in the right place. (Aizawa, 2010b, p. 271)

The experiment by Sur and his colleagues provides evidence for the plasticity of neuronal setting, but says nothing about the extension of the focus of investigation. Although it provides evidence supporting theories which propose that surgical intervention changes neuronal activity in different brain areas as regards different sense modalities, it does not do so for the claim that the brain is exclusively the right place for an explanation of, e.g., the visual character of an experience.

Beyond the difficulties of correct interpretation and abandoning too-demanding conclusions, we face arduousness posed by philosophers. Watson referred to the linguistic turn, and thanks to this we are prepared at least to notice some of the grammatical or logical and categorical mistakes. But if we go back a bit further to the late $19^{\text {th }}$ and early $20^{\text {th }}$ century, we can find unnoticed indications of confusion in philosophy. As I have previously mentioned (Kondor, 2015), Bergson emphasized the peculiar character of our concepts, while James indicated that we are inclined to duplicate different phenomena when we forget that the "thought-of-an-object, and the object-thought-of" are the same.

\section{Consciousness: Neuronal-Based vs. Embodied and Enacted}

At the turn of the $20^{\text {th }}$ century, consciousness was considered as a crucial element in understanding psychic or mental phenomena. With his insights regarding memory, attention, consciousness, etc., William James is regarded by many researchers to be one of the "founding father[s] of modern psychology" (Dehaene, 2014, p. 12; Baars, 1988, p. 13). His idea of an "empirical parallelism" between "the succession of states of consciousness with the succession of total brain-processes" (James, 1932, p. 182) is appreciated even today. However, as we will see, some of his convictions provide grounds for criticism as well (Baars, 2011, p. 13f.).

During the $20^{\text {th }}$ century there was considerable, though diverging, progress in cognitive studies and research into the mental life. After decades of the dominance of behaviourism in psychology, consciousness again entered the focus of interest thanks to technical developments. Thanks to brain imaging technologies, psychology, whose earlier methods were 
considered subjective because of a lack of repeatable measuring, was provided with instruments which offered a link between subjective reporting and measurable brain activity (Frith, 2007, p. 12). However, this link seems to be a faulty one since it can only yield data about correlation, but is not capable of affording evidence for causation or constitution. This would require other, invasive experimental techniques, such as local stimulation of brain areas and/or neurons (Dehaene, 2014, p. 180ff.).

In the first half of the $20^{\text {th }}$ century, philosophy suggested that meaning is possible only against the background of consciousness; that is, the higher order cognitive processes and faculties that make us unique among living creatures are due to consciousness. If we consider recent findings, we can see that consciousness is mostly comprehended as a capacity that provides a better chance for survival and makes higher order cognitive functions possible.

In what follows, I will attempt to focus on two radically different approaches to consciousness. Specifically, I will outline theories that seek a neural correlate of consciousness and are aimed at creating a causal model of neuronal activity and conscious experience, and I will also delineate approaches which suggest consciousness can be understood only in a wider setting, including the body and its environment.

\section{Focusing on Intracranial Processes}

Most of the time when consciousness is in focus, we are reminded of the old metaphysical question of how to relate physical and mental phenomena. The question has been reformulated in various ways, but even now many consider it a source of considerable difficulty. "Although neuroscience has identified many empirical correspondences between brain activity and mental life, the conceptual chasm between brain and mind seems as broad as it ever was" (Dehaene, 2014, p. 190). However, Bernard J. Baars, the originator of a very influential theory, proposed that we avoid asking it entirely.

In his book titled A Cognitive Theory of Consciousness (1988), Baars laid the foundation of global workspace theory (GWT). Baars suggests we disregard the old metaphysical question in favour of considering consciousness as a theoretical construct, in the same way as atoms were regarded a long time ago, as gravity was later, and not that far in the past genes, which were described based on inference rather than observation. (Baars, 2003, p. 2ff.). An additional requirement of this perspective is to find observable and measurable states as they relate to consciousness, and contrast them with unconscious ones. We need to operationally define that which can be considered as being conscious. We must also be able to distinguish conscious states from other related theoretical constructs such as attention, memory, executive processes, etc. We can find four conditions of conscious processes which fit well into standard research practice: they "1. can be reported and acted upon, 2. with verifiable accuracy, 3. under optimal reporting conditions, 4. and which are reported as conscious" (Baars, 2003, p. 4). Following the contrastive method and taking into consideration the abovementioned operational criteria, global workspace theory can be described in an unambiguous way. It "is a cognitive architecture with an explicit role 
for consciousness in humans. It suggests that conscious contents involve a fleeting integrative memory capacity in the brain, in which only one consistent content can be dominant at any given moment" (Baars \& Gage, 2010, p. 287).

Stanislas Dehaene's global neuronal workspace theory, while accepting the core idea of GWT, i.e., that consciousness is a global broadcasting of information within the cortex, suggests consciousness "arises from a neuronal network whose raison d'être is the massive sharing of pertinent information throughout the brain. ...We believe that a special set of neurons diffuses conscious messages throughout the brain: giant cells whose long axons crisscross the cortex, interconnecting it into an integrated whole" (Dehaene, 2014, p. 24f.). In accordance with the original theory, he also devotes considerable attention to unconscious processes which can be considered as being on the one hand automatic and reflexive functions, and which, importantly, can yield an informational background as a possible source of intuition on the other hand. The unconscious plays an important role in various capabilities, such as word comprehension, numerical addition, error detection, and problem solving - just to name a few. It operates quickly and in parallel across an extensive range of stimuli and responses (Dehaene, 2014, p. 106). Prior studies had recognized that damage in certain brain areas can cause strange constellations of normal function and dysfunction, such as blind sight, visual form agnosia, and spatial neglect ${ }^{3}$ - to mention a few well discussed cases where unconscious perception undoubtedly plays an important role in the patient's behaviour. Experiments that tested binocular rivalry and attentional blink provided evidence that shows there is a persistent fight for conscious access (Dehaene, 2014, p. 42). ${ }^{4}$

Experiments with masking revealed that unconscious processing can facilitate or bias conscious responses. It turned out there is a well-defined threshold whereby visual stimuli are consciously perceivable or not: when an image is presented for 40 milliseconds it is utterly invisible, but it is readily noticeable when the duration is increased to 60 milliseconds (Dehaene, 2014, p. 54).

An experiment that tested intuitions regarding probability and numerical analysis conducted in 1997 (and reinforced in 2011) nicely showed the effect unconscious processing

\footnotetext{
${ }^{3}$ In the case of blind sight lesion in the primary visual cortex, conscious vision is rendered impossible (patients report they do not see, let's say a flash of light, but can accurately point to the place of the flash); patients suffering from visual form agnosia are not able to recognize form, but their motor system behaves as if the patient could recognize it; we speak about spatial neglect if, e.g., the lesion is near the right inferior parietal lobe and the patient ignores the space on her left side and is impaired in her conscious judgements, though not really blind.

4 "Binocular rivalry reveals a competition between two simultaneous images. During the attentional blink, a similar competition occurs across time, between two images that are successively presented at the same location" (Dehaene, 2014, p. 45).
}

We need to note here that Dehaene considers conscious access as a key condition for consciousness. "What counts as genuine consciousness, I will argue, is conscious access - the simple fact that usually, whenever we are awake, whatever we decide to focus on may become conscious. Neither vigilance nor attention alone is sufficient."(Dehaene, 2014, p. 18) As we will see, his suggestion is consonant with others' considerations. 
may have in decision making. ${ }^{5}$ The experiment seems to provide evidence that we may have advantage of an unconscious incubation period. Since conscious processing entails a huge load on working memory and only one thing at a time can be consciously processed, it takes considerable time to calculate from random choices which alternatives yield a better gain with more security. At the same time, "[u]nconscious processes excel in assigning values to many items and averaging them to reach a decision" (Dehaene, 2014, p. 101).

The importance of unconscious processing and even unconscious perception is beyond question. Chris Frith assigns to the unconscious a curious, but significant role, viz., our brain provides us with illusions. These illusions suggest, among other things, that there is a separated physical and mental world, that we have direct access to the objects of the external world, and that we bear an isolated and private mental world.

As we can see in masking experiments and the above-described case of unconscious prediction, we can easily accept Frith's suggestion that "our brain doesn't tell us everything it knows." But he goes further and proposes our brain sometimes "actively misleads us" (Frith, 2007, p. 47). Visual illusions (Frith refers to Hering lines, the Pantheon illusion, and the Ames room) and the bottleneck of conscious processing underpin this suggestion. Taking into account the Ames room, we see three men who are extremely different in size (as opposed to the other possibility, which is in fact the foundation of the illusion, a modified, not normally shaped room). How does our brain interpret and decide which interpretation to choose on the basis of "the very limited and imperfect signals provided by our senses" (Frith, 2007, p. 85)?

Correctly predicting what comes next is crucial for survival. Predictive skills are learned capabilities and, according to Frith, our brain learns in accordance with the rules of associative learning. As Pavlov's dog, Thorndike's cats, and Skinner's superstitious pigeons show, a pattern of behaviour that can be described in terms of prediction develops on the basis of trial and error plus reward and punishment. If after a given signal or action the reward emerges in a short time, subjects assign high value to it; otherwise the signal or action will not be considered as being worthy of interest. The correctness of predictions is affirmed or disaffirmed by unconscious chemical processes in the brain. Nerve cells release neurotransmitters, and these neurotransmitters are important indicators of fitness. Accordingly, the nerve cells' activity, i.e., whether they send a positive signal (unexpected reward), a negative one (expected reward does not arrive) or no signal at all (the prediction was correct), helps the brain to learn in a manner similar to what associative learning describes.

\footnotetext{
${ }^{5}$ In the experiment, subjects were given four decks of cards and a symbolic loan of $\$ 2000$ to manage. On each card there was a message stating that they had earned or lost $\$ 100$. Two of the decks were composed to earn a lot at the beginning but lose heavily later on, whilst the other two provided a balanced, but not too high, gain. The time period when subjects randomly choose among the decks without any preconception or calculation was the focus of the experiment. "Fascinatingly, just before they choose a card from a bad deck, their hands begin to sweat, thus generating a drop in skin conductance. This physiological marker of the sympathetic nervous system indicates that their brain has already registered the risky decks and is generating a subliminal gut feeling." (Dehaene, 2014, p. 100)
} 
This learning process entails probability. As the McGurk effect ${ }^{6}$ shows, our brain combines different sense modalities, and when it receives contradicting information, it tries to fuse them together and give one unambiguous percept. That is, we move in a world which is reconstructed by our brain in accordance with certain predictive capabilities and permanent checking. We have no access to most of this reconstruction process because these processes are not conscious. Other people's behaviour and intentions are reconstructed in the same fashion. Similarly, we do not control our body in the manner we believe we do. We are only aware of our goals and preferences but not the motor commands, the efferent and afferent copies which provide smooth execution.

Frith suggests "“I do ...' indicates those situations where I am aware of what my brain is doing. But the 'I' in this case is still my brain." (Frith, 2007, p. 106) And moreover, the border of my body blurs, just as the imagined and believed self is modified in order to understand others. "Your prejudices and your observations of their [others'] behavior automatically make you become, for a moment, more like the person you are interacting with. This makes it easier for you to predict what they will do or say next" (Frith, 2007, p. 170).

As we can see, neural-based reconstructions of conscious and unconscious processes suggest conscious states are produced strictly in the brain. We can see correlations between mental states and neural activity, and we can describe brain's activity as a learning process based on earlier experiences and certain chemical processes in the brain. But we need to ask: How can the brain as predictive machinery learn without external stimuli? Descriptions of different experiments and the acquired results seem to suggest that the investigation is designed to be able to prove that neural mechanisms are the cause of conscious states. That is, we presuppose that intracranial processes are responsible both for conscious and unconscious processes.

Beyond these suggestions, there are arguments which seemingly support the proposal that consciousness is the result of pure brain activity. The extensive literature on phantom limb pain seems to provide evidence that the brain in itself is capable of creating an illusion of pain, or non-existent stimuli on non-existent limbs. Voluntary actions also seem to be a good candidate for evidence that brain is an autonomous driver of behaviour. According to Libet's experiment, "brain activity indicating that the volunteer was about to lift a finger occurred about 300 msec before that volunteer reported having the urge to lift his or her finger" (Frith, 2007, p. 66). That is, our brain starts to arrange the planned movement before our conscious report. ${ }^{7}$ Hence, our brain plays a decisive role in voluntary actions, and moreover, our brain makes an unconscious choice before we become aware of it (Frith, 2007, p. 68).

\footnotetext{
${ }^{6}$ McGurk's experiment demonstrates how our brain combines visual and auditory perceptions. The movement of the lips suggest ga ga ga, the mere sounds suggest $b a b a b a$, and when we see and hear simultaneously, we hear da da da.

${ }^{7}$ Here I would like to call your attention to Ramachandran's remark according to which, "Perhaps our very notion of causation requires a radical revision ....as happened in quantum mechanics" (Ramachandran, 2004, p. 89).
} 
Our brain is capable of deluding us, according to Frith, by suggesting truth underlying the illusions of the distinctness of mental and physical, direct access to the things of the world, and a separate private physical and mental life that is under control. That is, our brain is capable of framing our mental setting due to its unconscious processes. Since neural activity shows a pattern of connectedness in our brain, investigating neural networks and connectivity may provide a code which illuminates how our brain transforms our thoughts. Although "[w]e still do not understand exactly how millions of neuronal discharges, distributed across time and space, encode a conscious representation," we do not give up trying to find "new mathematical instruments in order to understand these complicated patterns" (Dehaene, 2014, p. 164f.).

Beyond illusions, including phantom sensations and our belief in voluntary actions, there is an additional aspect underlying the brain's exhaustive role in consciousness, namely the brain's autonomous functioning. "Autonomy is the primary property of the nervous system. Intrinsic neuronal activity dominates over external excitation. As a result, our brain is never passively submitted to its environment but generates its own stochastic patterns of activity" (Dehaene, 2014, p. 222). However, a question may be asked: If we consider continuous background electrical oscillations as being a facilitator of the synchronization of the cells, how can we consider the maintenance of it as autonomy against the background of an evolving brain structure which adapts to more and more complicated tasks?

Similarly, we may have doubts whether patients who have never had a certain limb could have the experience of that phantom limb or phantom pain in it. Likewise, if we take into consideration that conscious processing is time-consuming as compared with non-conscious processing, why is it surprising that the conscious report is delayed compared to the onset of cerebral action?

Regarding the autonomy of the brain when consciousness is in question, we have a huge amount of data on how ongoing oscillation supports smooth processing, as well as how consciousness ceaselessly stabilizes perception, creates lasting thoughts, and sometimes illusions and phantom pains. We have different models of how consciousness is beneficial for biological success (Humphrey, 2000; Grazianom 2014) and how it creates a virtual world (Frith, 2014). But although in each case it is not explicitly proposed that the brain is separate from its environment, it is suggested that neural activity is the key to consciousness. Notwithstanding, I find it peculiar to argue for the brain's autonomy whilst considering adaptation and learning as decisive in both the brain's phylogeny and ontogeny.

\section{Consciousness Enframed}

Hereinafter, I will give a rather rough outline of slightly different approaches that are related to embodiment and enactment and which view consciousness from a different angle, giving it a more extended basis than the previously delineated ones. 
The notion of embodiment has been explicated by Maurice Merleau-Ponty in his 1945 book, Phénoménologie de la perception. He suggests "far from my body's being for me no more than a fragment of space, there would be no space at all for me if I had no body" (Merleau-Ponty, 2005, p. 89); and a couple of pages later: "if the words 'enclose' and 'between' have a meaning for us, it is because they derive it from our experience as embodied subjects. In space itself independently of the presence of a psychophysical subject, there is no direction, no inside and no outside" (p. 182).

This idea is revived in many fields, including art history thanks to Rudolf Arnheim, cognitive metaphor theory as a result of work by Mark Johnson and George Lakoff, and more importantly from our perspective, "in new sciences of the mind" (Varela et al., 1993, p. xv). Merleau-Ponty's holistic view suggests transforming the dualism of the soul and body into the distinction of "the lived and the known." It is possible because a human being, the so-called subject, "lives in a universe of experience, in a milieu which is neutral with regard to the substantial distinctions between the organism, thought and extension; he lives in a direct commerce with beings, things and his own body" (Merleau-Ponty, 1963, p. 189). In his view, consciousness provides the ground for a meaningful unity where the ambient world, the acting subject and its body, thus the mental and the physical, are inseparable. In accordance with Merleau-Ponty's views, the term embodiment suggests "that cognition depends upon the kinds of experience that come from having a body with sensorimotor capacities, and ...that these individual sensorimotor capacities are themselves embedded in a more encompassing biological, psychological, and cultural context"(Varela et al., 1993, p. 172f.). Theories built upon the idea of embodiment vary depending on how they relate to computationalism, mental representation, and to what extent they consider its validity. For now I will not go into details regarding the differentiation of the notion of embodiment; rather, I will focus on different conceptions which suggest consciousness and cognitive processes are inseparable from having a body, bearing bodily skills and interactively being immersed into our environment.

We can find many fruitful attempts in the last centuries of the history of philosophy that call attentions to the importance of the body (James), movement (Bergson), our embeddedness (Heidegger), and environment as it plays a role in our consciousness (MerleauPonty). All these suggestions relate in some way to the metaphysical question of dualism: these theories attempted to eliminate or a least reformulate the body-mind divide, but take significantly different routes from what Baars suggested. We already touched the question of dualism when referring to Baars' suggestion that we need to abandon the metaphysical burden of body-mind dualism and in hinting at his criticism of James' doubts (Baars, 1988, p. 14). I believe that James' scruples about consciousness' ontological status were not baseless. First, in his 1904 paper he calls into question only that consciousness "stands for an entity" but still believes that "it does stand for a function" (James, 1987, p. 1141f.; emphasis added). GWT is also looking for function: the distinctive criteria of consciousness are functional ones and, more precisely, the neural patterns entail a certain capability, function, but not an entity. 
At the same time, GWT is tangled up unnoticed with the metaphysical question of bodymind dualism when it tries to demonstrate a causal, sometimes constitutional, relation between brain processes and mental states. When searching for the neural correlate of consciousness or trying to illuminate the causal relation between subjective reports of phenomenal experience and brain activity, I believe it is illuminating to recall James' caveat that "any single non-perceptual experience tends to get counted twice over, just as a perceptual experience does, figuring in one context as an object or field of objects, in another as a state of mind: and all this without the least internal self-diremption on its own part into consciousness and content" (James, 1987, p. 1148).

Against the background of James' neutral monism, this duplication of the same phenomenon is avoidable. He considers pure experience as the only "primal stuff or material in the world, a stuff of which everything is composed." If we take pure experience as our departure (as opposed to the dualism of physical and mental), the relation between the subject and object "is a part of pure experience; one of its "terms" becomes the subject or bearer of the knowledge, the knower, the other becomes the object known" (James, 1987, p. 1142).

This monism may remind us of recent efforts in certain enactive and sensorimotor approaches. The enactive approach emphasizes "that cognition is not the representation of a pregiven world by a pregiven mind but is rather the enactment of a world and a mind on the basis of a history of a variety of actions that a being in the world performs" (Varela et al., 1993, p. 9).

According to Evan Thompson, the enactive approach tries to unify the idea of autonomous agents as they generate and maintain themselves and thus their cognitive domain: the nervous system is considered as a dynamic system which creates meaning; cognition as being "the exercise of skilful know-how in situated embodied action"; the world is conceived as being a relational domain enacted; and experience is conceived as crucial in the understanding of the mind and has to be investigated phenomenologically (Thompson, 2007, p. 13). Against the background of the above-sketched scenario, the "transformation of the world into an environment happens through the organism's sense-making activity." Accordingly, cognition does not happen internally but rather it "is a relational process of sense-making that takes place between the system and its environment" (Thompson \& Stapleton, 2009, p. 25f.). Since cognition is defined as a relational process, the cognizing agent and its environment are not separable. Additionally, the coupling between them is without any gap, because this cognizing agent is a physical system which therefore has direct access to its physical environment.

Radical branches of the enactive or sensorimotor approach make similar efforts to illuminate their radicalism, which is made of a kind of monism and dynamism. There is an important difference between the abovementioned enactive approaches and the subsequently outlined ones: the latter attempt to avoid the use of terms which entail reasoning, a demanding higher order cognitive function. On this basis, the so-called hard problem of consciousness is not solvable, but meaningless, and representationalism is radically mitigated. 
Radical embodied cognitive science (Chemero, 2009), or more recently extended cognitive-phenomenological system theory (ECPSt; Silberstein \& Chemero, 2011, 2015) take the point of James' neutral monism, dynamic system theory, and J. J. Gibbson's account of affordance. ECPSt considers cognitive systems as being "extended brain-body-environment systems." But they can be regarded as being extended only "when environmental features form constitutive parts of the cognitive phenomenon" (Silberstein \& Chemero, 2015, p. 189). Since experience is processed cognitively and cognition is experiential, the "phenomenological world of experience is neither in the 'head' nor in the 'external world' - it is fundamentally relational" (p. 190). According to ECPSt - unlike Frith and Dehaene- "the brain is not some sort of virtual reality machine that generates a matrixworld internal to the brain. The very idea of a neural correlate of consciousness as a sufficient condition for some conscious state is a misnomer" (p. 190).

With similar targets in mind but a slightly different focus, Hutto and Kirchhoff recapitulate the attempt, abandoning content-involving basic cognition. As they wrote:

In Radicalizing Enactivism, Daniel Hutto and Erik Myin (2013) champion a vision of enactivism according to which the plethora of cognitive activity of humans and non-human organisms is best explained in terms of and understood as dynamically unfolding, situated embodied interactions and engagement with environmental affordances. Radical embodied-enactive approaches to cognition (REC, for short) thus reject the familiar assumption that the best explanation of cognition always requires positing contents that are acquired and transformed in order to create representations that then inform and guide what an organism does or experiences. (2016, p. 348)

That is, REC takes its departure, like ECPSt, by considering the cognizing agent, its bodily conditions and environment, as a dynamically intertwined system which does not need mental representations while it is engaged in and with its environment. This idea had already re-emerged in 2004: "why an internal representation would be any better than access to the world itself. This harkens back to Wittgenstein's idea that anything a picture in the head could do could be done by a picture held in the hand. We go a step further: Why do we need a picture at all? The world is right there, after all. We are in the world" (Noë, 2004, p. 218f.).

Both the REC and ECPSt approaches attempt to abandon mental representation based on the above quoted Wittgensteinian considerations on the one hand and the Jamesian caveat on the other. Both REC and ECPSt suggest that the hard problem is unsolvable, and that therefore there is no need to disprove it, but both provide a framework within which it has no sense. That is, phenomenality "should be understood as the character of engaging with the world in different ways" (Hutto \& Kirchhoff, 2016, p. 353). 
The so-called sensorimotor approach, agreeing with the importance of active engagement when having a feel, or having a conscious experience ${ }^{8}$ bearing a certain quality, goes a bit further when it suggests that "what constitutes the fact of having a feel is that changes like those I have just listed ${ }^{9}$ will occur when we do certain thing. ... [T] his state of affairs constitutes the feel" (O’Regan, 2011, p. 158).

As we can see on the basis of earlier philosophical considerations, the enactive and sensorimotor approaches suggest that cognitive processes and, specifically, consciousness and its phenomenal character are hardly comprehensible as being exclusively intracranial. It is quite obvious that the theories referred to in the earlier section of this paper are more or less committed to a different framework than the latter ones. The former are based on a vocabulary that reaches back to cognitive psychology, which is attached to representationalism, and importantly, to searching for neural mechanisms when consciousness and different cognitive skills are in question. The latter theories are committed to abandoning representationalism and taking the perspective of a rather holistic and dynamic approach to cognitive processes. In what follows, I will attempt to show that despite the difference between the frameworks and presuppositions, the seemingly unbridgeable gulf between the descriptions can at least be attenuated.

\section{Representation Revisited}

Authors, even those who think the brain is the right place to look if we are seeking an explanation of the modal character of a perceptual experience, express suspicion regarding the tight commitment to representations: "it is unclear just how completely cognitive states must be representational. ...Must every component of every cognitive state be a representation? This seems to be an overly strong empirical hypothesis not warranted by any data we know of." (Adams \& Aizawa, 2010a, p. 55)

Regarding the scope and limits of representations, Adams \& Aizawa formulate an additional concern:

We would not want to claim that these neurons [in different visual areas which participate in creating the visual field of an organism] are representations simply in virtue of their causal connections to environmental stimuli, but we do think they are likely to turn out to be representations. These considerations seem to us to provide defeasible reasons to accept what cognitive psychologists typically presuppose, namely that cognitive processes involve representations. (2010a, p. 33)

\footnotetext{
${ }^{8}$ For a short outline of O'Regan's conception of conscious access and phenomenal consciousness see Kondor (2015, p. 153f.). For further details see O'Regan (2011).

${ }^{9}$ A non-exhaustive list of the kinds of laws which apply when a part of one's body is touched: "Active motion of the body part" modifies the stimuli; "active motion by a different body part" in special cases can alter the stimuli; "passive motion" induced by external force changes the stimuli; at the same time we perceive change in our visual field; and in some cases we can hear temporally correlated sound of scratching (O’Reagan, 2011, p. 157).
} 
This suspicion illuminates the ambiguous character of representation as it is used in psychology, and also in neurosciences as we will see. According to traditional cognitive science, representations play a role in perception, remembering, reasoning, and dreamingto mention only a few important mental activities. Cognitive science maintains that representational states are content-bearing and carry information in a certain way. We perform operations over them and these operations result in problem-solving and planning. Representations presuppose certain relations, such as mapping, (i.e., environmental structures are coded onto internal structures in accordance with certain rules); intentionality, since representations are about something; asymmetry between the representation and the represented; and standing-in relation (i.e., that which a representation stands for is the representation's content; Nagel, 2005, p.5).

Richard A. Watson suggests that representations are considered from the very beginning as they are based on isomorphism. Isomorphism is meant to be "any degree or kind of resemblance, likeness, or similarity of pattern, structure, or relational organization between entities or events as defined in the broadest sense" (1995, p. xi). Even authors like Descartes, who denied the necessity of resemblance (in the above-described sense) between the represented entity and its idea, fall back to the route of isomorphism. Watson's argument shows that Descartes' ambiguity is rooted in his ontological commitment to the dualism of the extended and cognising substances, and his clarifying efforts often fail because the linguistic toolkit imposes an extra burden when trying to close the gap between two substances.

Beyond ambiguities, Watson quotes Descartes calling attention to an important distinction within a dualist framework:

The subject I propose to deal with in this treatise is light, and the first point I want to draw to your attention is that there may be a difference between the [i] sensation we have of light (i.e., the idea of light which is formed in our imagination by the mediation of our eyes) and [ii] what it is that produces this sensation within us (i.e., what it is in a flame or the sun that we call by the name "light"). (1995, p. 30)

Descartes seemed to be very distinctive when he calls our attention to two different phenomena when we attempt to understand the same process. That is, (i) the sensation of light which is mediated by our eyes and processed further in a way ${ }^{10}$ and (ii) what is the cause of our sensation. This distinction gains special emphasis against the background of James' previously mentioned caveat, and, at the same time, is very important in light of the question of whether we can limit the investigation of conscious experience to brain mechanisms or should extend it to the interaction between the organism and its environment.

Nowadays, thanks to brain imaging techniques, neural activity is considered as a representation of a certain mental state. In this case, a mental state is represented via neural activity, i.e., the neural state is considered a physical equivalent of a mental phenomenon. On the basis of representation as based on isomorphism, Dehaene's proposal that we can

\footnotetext{
${ }^{10}$ It is beyond the framework of the present paper to discuss how ambiguous Descartes' notions of imagination and conception are regarding representation. For details, see Watson (1995, pp. 19-37).
} 
detect patterns of active and inactive cells as they are forming "an internal code for the contents of subjective perception" (2014, p. 176) is promising. But, as Dehaene himself notes, "[p]roving that a pattern of brain activity causes a mental state is one of the hardest problems facing neuroscientists" (p. 179). This is not mere bad luck, but rather it is rooted in the relation labelled as representation. When it is out of concern that a living organism is not reducible to its neural activity but is rather in a symbiotic relation with its environment, the fallacy of obliterating the difference between representation and causation can enter the scene. On the basis of correlation, we can suppose that neural activity, as we have access to it, represents a certain mental state, but it is hard to imagine that neural activity alone creates this state. Of course, there is no experiment which denies the existence of the stimuli, but since the scope of investigation is limited to brain processes, interaction with the body and the environment is inconspicuous and can easily fade into oblivion.

Nevertheless, the representation of neural activity via brain imaging techniques is visible, and is only a part of the process. The other part is the external stimuli: the flame or the sun. If we take exclusively intracranial processes into consideration, it will be challenging indeed to provide causal relations. Cause and representation requires different time orders: the cause is prior to its effect; a representation is a representation of something that exists in a certain way before its representation. ${ }^{11}$ Accordingly, if we consider neural activity as being a cause of a certain mental state (e.g., consciousness), it cannot be considered as being its representation. The visible reconstruction (publicly available representation) of a neural state (thanks to brain imaging and statistical methods), I believe, does not provide satisfactory grounds for thinking that this neuronal constellation generates the given mental states.

It is beyond question that if we are talking about mental representation, we need to understand it as a theoretical object. But often, representation is not appropriately specified. Public representations which are accessible to others are not theoretical constructs, but artefacts. From this perspective, it is quite clear that the representation of a neural state (which is considered as being in correlation with a mental state) can cause anything in the subject's behaviour.

A representation is a theoretical object that bears an abstract resemblance to something outside itself. In somewhat different terms, there is an abstract match or isomorphism between the representation and the thing that is represented....We can think of knowledge, percepts, images, plans, intentions, and memories as representations.... What is the adequate evidence for the existence of a mental representation? ${ }^{12}$ In psychology we often infer that human beings have mentally represented an object if they can correctly detect matches and mismatches to the object at a later time. (Baars, 2011, p. 41)

\footnotetext{
${ }^{11}$ Haptic images, which are similarly interactive as virtual ones but they "were to be manipulated as part of the modification or construction of a physical object, as in nanomanipulation" (Daston \& Galison, 2007, p. 385), may confuse this time order. But cognition as a brain-based activity does not necessitate going into detail regarding how the manipulation of representations entails.
}

${ }^{12}$ Cf. Adams' \& Aizawa's doubts (2010a, p. 55). 
The above description is clearly about mental representation. The following one refers to public representations, but the point of departure is not that unambiguous: "Ideas appear to be represented in the cortex in terms of complex webs of learned connectivities, rather than localized filing systems with neatly arranged conceptual categories" (Baars \& Gage, 2013, p. 360; emphasis added). As with Dehaene's description of a conscious percept, we have a pattern of neural activation of an idea, whatever it is.

"When I look at a tree in the garden, I don't have the tree in my mind. What I have in mind is a model (or representation) of the tree constructed by my brain. This model is built up through a series of guesses and predictions"13 (Frith, 2007, p. 170). The model of a tree as a mental representation is the result of the model-making brain's learning process, i.e., it is supposed to be generated by the brain. "The fortress of the conscious mind possesses a small drawbridge that forces mental representations to compete with one another" (Dehaene, 2014, p. 46). This lively description of the bottleneck of consciousness suggests we have unconscious mental representations competing to become a conscious one. But it does not help us find out what we should think about these unconscious mental representations. Unconscious mental representations are not accessible subjectively; accordingly, when we are to detect them we can have only indirect reports about them. Perhaps it is even more puzzling when Dehaene describes the working of certain specialized neurons: single neurons which can be activated by a definite picture, sound, etc., but only in the case of conscious perception (p. 172f.). Conscious perception is, at the same time, a special neural network activation within which the communication between different brain areas, even in a hierarchical sense, is highly active and bi-directional. If we suppose a causal relation between neuronal state and conscious perception, where can we find the mental representation of the percept: in the communication of the network, or in the single neuron which is sensitive to a given phenomenon?

The above outlined picture can be summarized as follows: The term representation is used at least in two distinct senses: (i) in the sense of neural pattern, i.e., as a mental event described in terms of neuronal activity; and (ii) in the sense of the content of consciousness (Frith, 1999), or as a theoretical construct that stands for a mental phenomenon (Baars, 2011). ${ }^{14}$ In the first case, representation is publicly available, i.e., we have access to neural states via brain imaging technologies. In the second case, the content of consciousness and/or the mental phenomenon is merely presupposed, as it occurs in the brain and we hardly have any access to them at the moment. Unfortunately, we cannot yet crack the code of how the brain creates a mental state (Dehaene, 2014) and we do not know any physiological marker which can indicate that a mental representation has been formed (Frith, 1999). Importantly, we have no evidence that cognitive processes involve mental representation, it is merely presupposed. Additionally, we do not know to what extent a cognitive

\footnotetext{
13 The idea of brain as a "model-making machine" is rather accepted. See also Ramachandran (2004, p. 105).

14 The content of conscious experience and different mental phenomena can be considered interchangeably since Baars' listed examples are possible only in case of conscious processing.
} 
state should be representational as regards its components. We have direct access only to the scenery, behaviour, report, and recorded neural states. In accordance with this information, mental representation was introduced as a shortening of what could be the content of the conscious experience and/or cognitive state of which we can find the neural correlate.

But if we keep in mind what James suggested, it easily turns out that mental representation is only a presupposed duplicate of the perceived scenery that we should have found in neural activity. Introducing mental representation when we search for the connection between the object of one's conscious experience and the physiological changes in our nervous system entails more difficulties than answers. The introduced theoretical construct creates difficult to solve questions: what kind of physiological marker should we seek to be able to detect when a mental representation is under construction? How can our brain capacity adapt to permanent change regarding its representational burden? What is the difference between the neural pattern of a cognitive state and its content?

Mental representation as models, or webs of learned connectivities which are capable of competition, mirrors the presupposition of the enterprise, viz., to prove that our brain generates consciousness, hence the phenomenal character of experience. This means we have to accommodate the whole body and its experiences in the brain in a way that allows us to gain answers to the questions phenomenal experience raises. If we work within an embodied and enacted framework and accept that the sensorimotor approach suggests that experience is not generated somewhere, rather that it is a capacity to interact with the world, then we will not need to find the content of consciousness in the brain.

\section{Conclusion}

In conclusion, I would like to draw attention to the curious situation where although theoretical constructs may aid inquiry and metaphoric language may help expression and the explication of a new phenomenon, they are also likely to engender confusion. If, on the one hand, representation is used without strict distinctiveness between mental and public and we are forgetful about mental phenomena as a theoretical construct, we may be tangled up with unsolvable paradoxes and concealed gaps. On the other hand, taking radical views on enactment and embodiment into consideration, specifically their effort to abandon representationalism and consequently its vocabulary, we can remove the obstacles from the route of clarification of how behaviour and organism, including its environment, are intertwined.

\section{References}

Adams, F., \& Aizawa, K. (2010a). The bounds of cognition. Malden, MA: Wiley-Blackwell.

Aizawa, K. (2010b). Consciousness: Don't give up on the brain. Royal Institute of Philosophy Supplement, 67, 263-284. doi:10.1017/S1358246110000032 
Baars, B. J. ([1988] 2011) A cognitive theory of consciousness. Kindle edition published by the author.

Baars, B. J (2003). Treating consciousness as a variable: The fading taboo. Baars, B. J., Banks, W. P. \& Newman J. B. (Eds.), Essential sources in the scientific study of consciousness. Cambridge, MA: The MIT Press.

Baars, B. J., Franklin, S., \& Ramsoy, T. Z. (2013). Global workspace dynamics: cortical "binding and propagation" enables conscious contents. Frontiers in Psychology, 4, 1-22. doi: 10.3389/fpsyg.2013.00200

Baars, B. J., \& Gage, N. M. (Eds.). (2010). Cognition, brain, and consciousness. Oxford, UK: Elsevier.

Baars, B. J. \& Gage, N. M. (2013). Fundamentals of cognitive neuroscience. Amsteram, the Netherlands: Elsevier.

Baars, B. J., Seth, A. K., \& Edelman, D. B. (2005). Criteria for consciousness in humans and other mammals. Consciousness and Cognition, 14, 119-139.

Chemero, A., \& Silberstein, M. (2012). Complexity and extended phenomenological-cognitive systems. Topics in Cognitive Science, 4(1), 35-50.

Daston, L. \& Galison, P. (2007). Objectivity. Cambridge, MA: MIT Press.

Dehaene, S. (2014). Consciousness and the brain. Deciphering how the brain codes our thoughts. New York, NY: Viking/Penguin Group.

Dennett, D. C. (1991). Consciousness explained. New York, NY: Little, Brown and Company.

Frith, C., Oerry, R. \& Lumer, E. (1999). The neural correlates of conscious experience: an experimental framework. Trends in Cognitive Sciences, 3(3), 105-114.

Frith, C. (2007). Making up the mind: How the brain creates our mental world. Oxford, UK: Blackwell Publishing.

Gallagher, S. (2000). Philosophical conceptions of the self: Implications for cognitive science. Trends in Cognitive Sciences, 4(1), 14-21.

Gallagher, S. (2005). How the body shapes the mind? Oxford, UK: Oxford University Press.

Gallagher, S., \& Zahavi, D. (2008). The phenomenological mind: An introduction to philosophy of mind and cognitive science. London, UK: Routledge.

Graziano, M. S. A. (2014). Speculations on the evolution of awareness. Journal of Cognitive Neuroscience, 26(6), 1300-1304.

Humphrey, N. (2000). How to solve the mind-body problem. Journal of Consciousness Studies, 7(4), $5-20$.

Hutto, D., \& Kirchhoff, M. D. (2016). Never mind the gap: Neurophenomenology, radical enactivism, and the hard problem of consciousness. Constructivist Foundations, 11(2), 346-353.

Hutto, D. D., \& Myin, E. (2013). Radicalizing enactivism: Basic minds without content. Cambridge, MA: MIT Press.

Jacob, P., \& Jeannerod, M. (2003) Ways of seeing? Oxford, UK: Oxford University Press. 
James, W. (1932). The principles of psychology: Vol. I. New York, NY: Henry Holt and Company. (Original work published 1890)

James, W. (1987). Does 'consciousness' exist? In W. James, William James: Writings 1902-1910 (pp. 1141-1158). New York, NY: Library Classics of the United States.

Knight, W. (2016, August 9). AI's language problem. MIT Technology Review. Retrieved from https://www.technologyreview.com/s/602094/ais-language-problem/

Kondor, Z. (2015). Theoretical controversies - terminological biases: Consciousness revisited. Studies in Logic, Grammar and Rhetoric, 41(54), 143-160.

Merleau-Ponty, M. (1963). The structure of behavior (L. A. Fisher, Trans.). Boston: Beacon Press.

Merleau-Ponty, M. (2005). Phenomenology of perception. (C. Smith, Trans.) London, UK: Routledge.

Nagel, S. K. (2005). Embodied, dynamical representationalism - Representations in cognitive science. Osnabrück, Germany: Institute of Cognitive Science.

Noë, A. (2004). Action in perception. Cambridge, MA: MIT Press

O'Regan, K. (2011). Why red doesn't sound like a bell? Oxford, UK: Oxford University Press

Polanyi, M. (1962). Personal knowledge: Towards a post-critical philosophy, London, UK: Routledge \& Kegan Paul.

Ramachandran, V. S. (2004). A brief tour of human consciousness. New York, NY: Pi Press.

Silbertstein, M., \& Chemero, A. (2015). Extending neutral monism to the hard problem. Journal of Consciousness Studies, 22(3-4), 181-194.

Silbertstein, M., \& Chemero, A. (2011). Complexity and extended cognitive-phenomenological systems. Topics in Cognitive Science, 4(1), 35-50.

Thompson, E. (2007). Mind in life: Biology, phenomenology, and the sciences of mind, Cambridge, MA: The Belknap Press of Harvard University Press.

Thompson, E., \& Stapleton, M. (2009). Making sense of sense-making: Reflections on enactive and extended mind theories. Topoi, 28, 23-30.

Varela, F. J., Thompson, E., \& Rosch, E. (1993). The embodied mind: Cognitive science and human experience. Cambridge, MA: MIT Press.

Wittgenstein, L. (1963). Philosophical investigations Oxford, UK: Basil Blackwell

Wittgenstein, L. (1980). Wittgenstein's lectures: Cambridge, 1930-1932. Chicago, IL: The University of Chicago Press.

Wittgenstein, L. (1979). Wittgenstein's lectures: Cambridge, 1932-1935. Oxford, UK: Basil Blackwell.

Wittgenstein, L. (1958). The blue and brown books. Oxford, UK: Basil Blackwell.

Zahavi, D. (2008). Subjectivity and selfhood. Cambridge, MA: MIT Press. 